\title{
Association studies of novel obesity-related gene variants with quantitative metabolic phenotypes in a population-based sample of 6,039 Danish individuals
}

\author{
K. S. Burgdorf • A. P. Gjesing • N. Grarup • \\ J. M. Justesen • C. H. Sandholt • D. R. Witte • \\ T. Jørgensen • S. Madsbad • T. Hansen • O. Pedersen
}

Received: 15 June 2011 /Accepted: 24 August 2011 / Published online: 28 September 2011

(C) Springer-Verlag 2011

\begin{abstract}
Aims/hypothesis Genome-wide association studies have identified novel WHR and BMI susceptibility loci. The aim of this study was to elucidate if any of these loci had an effect on quantitative measures of glucose homeostasis, including estimates of insulin release and insulin sensitivity in an epidemiological setting.

Methods By applying an additive genetic model, 14 WHRassociated gene variants and $18 \mathrm{BMI}$-associated variants were investigated for their relationships with glucoserelated metabolic traits in treatment-naive individuals from the population-based Inter99 study sample $(n=6,039)$.

Results Of the variants associated with BMI, the QPCTL
\end{abstract}

Electronic supplementary material The online version of this article (doi:10.1007/s00125-011-2320-4) contains peer-reviewed but unedited supplementary material, which is available to authorised users.

K. S. Burgdorf $(\bowtie) \cdot$ A. P. Gjesing $\cdot$ N. Grarup · J. M. Justesen •

C. H. Sandholt $\cdot$ T. Hansen $\cdot$ O. Pedersen

The Novo Nordisk Foundation Center

for Basic Metabolic Research, Faculty of Health Sciences,

University of Copenhagen,

Universitetsparken 1, DIKU Building, Room 1.1.N121,

DK-2100 Copenhagen, Denmark

e-mail: Kristoffer.Burgdorf@sund.ku.dk

K. S. Burgdorf $\cdot$ S. Madsbad

Hvidovre University Hospital,

Copenhagen, Denmark

D. R. Witte

Steno Diabetes Center,

Copenhagen, Denmark

T. Jørgensen

Research Centre for Prevention and Health,

Glostrup University Hospital,

Glostrup, Denmark rs2287019 C allele was associated with an increased insulinogenic index of $7.4 \%$ per risk allele $\left(p=4.0 \times 10^{-7}\right)$ and increased disposition index of 5.6\% $\left(p=6.4 \times 10^{-5}\right)$. The $L R P 1 B$ rs $2890652 \mathrm{C}$ allele was associated with insulin resistance, showing a $3.3 \%$ increase $(p=0.0011)$ using the HOMA-insulin resistance (HOMA-IR) index and a $2.2 \%$ reduction $(p=0.0014)$ with the Matsuda index. Of the variants associated with WHR, LYPLAL1/SLC30A10 rs4846567 G allele carriers showed a 5.2\% lower HOMA-IR $(p=0.00086)$ in women, indicating improved insulin sensitivity. Female carriers of the VEGFA rs6905288 A allele were insulin resistant, with a $3.7 \%$ increase in HOMA-IR $(p=0.00036)$ and $4.0 \%$ decrease in Matsuda index $\left(p=2 \times 10^{-4}\right)$.

T. Jørgensen $\cdot \mathrm{S}$. Madsbad $\cdot$ O. Pedersen Institute of Biomedical Science, Faculty of Health Sciences, University of Copenhagen,

Copenhagen, Denmark

T. Hansen

Faculty of Health Sciences, University of Southern Denmark, Odense, Denmark

O. Pedersen

Faculty of Health Science, University of Aarhus,

Aarhus, Denmark

O. Pedersen

Hagedorn Research Institute,

Gentofte, Denmark 
Conclusions Our correlative findings from analysing single-locus data suggest that some variation in validated BMI and WHR loci are associated with either increased or decreased insulin sensitivity and thereby potentially with metabolically healthy or metabolically unhealthy subsets of obesity. The results call for testing in larger study samples and for further physiological exploration of the possible metabolic implications of these loci.

Keywords BMI · Genetics · Insulin resistance . Insulin secretion · Obesity $\cdot$ WHR

$\begin{array}{ll}\text { Abbreviations } \\ \text { BIGTT-AIR } & \text { BIGTT-acute insulin response } \\ \text { GWAS } & \text { Genome-wide association studies } \\ \text { HOMA-IR } & \text { HOMA-insulin resistance } \\ \text { ISI } & \text { Insulin sensitivity index } \\ \text { SNP } & \text { Single-nucleotide polymorphism }\end{array}$

\section{Introduction}

Although the epidemic nature of obesity may predominantly be attributable to major changes in environmental factors for modern living, numerous genetic factors are known to predispose to altered body composition, including changes in BMI and WHR, both of which are inherited traits, with heritability estimates for BMI of $h^{2} \sim 40-70 \%$ and WHR of $h^{2} \sim 45 \%$ [1].

Since 2007, several genome-wide association studies (GWAS) have identified a number of common variants that are associated with BMI and WHR. Initially, variants in FTO and MC4R were reported to be associated with BMI $[2,3]$, and subsequent studies have revealed $>50$ loci associated with various aspects of adiposity $[1,4,5]$.

In 2010, two large GWAS meta-analyses from the GIANT (Genetic Investigation of ANthropometric Traits) consortium were published. Both studies conducted a discovery stage analysing $\sim 2.8$ million imputed and genotyped single-nucleotide polymorphisms (SNPs). In a study of BMI, 46 GWAS were meta-analysed including up to 123,865 individuals, and 42 were genotyped in stage 2 including up to 125,931 individuals. This study revealed 18 novel loci associated with BMI showing effect sizes on BMI of between 0.06 and $0.22 \mathrm{~kg} / \mathrm{m}^{2}$ per risk allele [4]. A parallel GIANT study investigated WHR adjusted for BMI in GWAS data from 32 studies comprising 77,167 participants with replication in a further 113,636 samples [5]. This study found 14 novel variants associated with WHR at a genome-wide significance level, with $\beta$ values between 0.022 and 0.043 per change in WHR-increasing allele. Sex-stratified analyses showed sex-specific effects, as 12 of 14 SNPs reached genome-wide significance in women and only three of 14 in men.

Given the hypothesis-free approach used in GWAS, most of the identified loci have no known involvement in pathogenesis of obesity or energy or glucose metabolism. No obvious biological mechanisms were identified for the BMI-associated variants; however, the GIANT investigators reported evidence that the WHRassociated loci may predominantly be associated with developmental processes, suggesting an important influence on basal body fat distribution [6]. This is in contrast with previous theories suggesting a major role for genes associated with body composition in regulation of appetite and energy expenditure [7-9].

Given the limited physiological knowledge of the biological implications of the recent variants discovered by the GIANT consortium, we aimed to elucidate in an epidemiological setting some of the underlying metabolic phenotypes of the 32 variants associated with either BMI or WHR, with a focus on estimates of insulin release and insulin sensitivity.

\section{Methods}

Study population The 18 SNPs associated with BMI and 14 SNPs associated with WHR were genotyped in treatmentnaive individuals from the population-based Inter99 study sample $(n=6,039)$. Inter99 is a population-based, randomised, non-pharmacological intervention study of middleaged individuals for the prevention of ischaemic heart disease, who were recruited through the Research Centre for Prevention and Health in Glostrup, Copenhagen (ClinicalTrials.gov ID-no: NCT00289237) [10]. The study participants underwent a standard $75 \mathrm{~g}$ OGTT. Patients with type 2 diabetes, type 1 diabetes or diabetes as a consequence of known chronic pancreatitis, haemochromatosis, mutations in the insulin receptor, lipodystrophy, maturity-onset diabetes of the young, maternally inherited diabetes and deafness, family history of first-degree relatives with type 1 diabetes, insulin requirement within the first year after diabetes diagnosis or a fasting serum C-peptide level $\leq 150 \mathrm{pmol} / 1$ at the time of recruitment were excluded from the study. All study participants were Danes by self-report, and informed written consent was obtained from all volunteers before participation. The study was approved by the regional ethics committees, Copenhagen County, and was conducted in accordance with the principles of the Helsinki Declaration.

Biochemical and anthropometric measures Body weight and height were measured with the participants wearing light indoor clothes and no shoes. BMI was defined as weight in kilograms divided by height in metres squared $\left(\mathrm{kg} / \mathrm{m}^{2}\right)$. Blood samples were drawn after a $12 \mathrm{~h}$ overnight 
fast. Plasma glucose was analysed by the glucose oxidase method (Granutest; Merck, Darmstadt, Germany), and serum insulin [excluding des(31, 32) and intact proinsulin] was measured using the AutoDELFIA insulin kit (PerkinElmer, Wallac, Turku, Finland). HOMA of insulin resistance (HOMA-IR), an index of hepatic insulin resistance in the fasting state, was calculated as (fasting plasma glucose $[\mathrm{mmol} / \mathrm{l}] \times$ fasting serum insulin $[\mathrm{pmol} / 1]) / 22.5$ [11]. The Matsuda index expressing whole-body insulin sensitivity index was calculated as described previously [12]. Low HOMA-IR and Matsuda index are associated with insulin resistance. The insulinogenic index was calculated as (serum insulin $30 \mathrm{~min}$ after OGTT [pmol/1]-fasting serum insulin [pmol/1])/plasma glucose $30 \mathrm{~min}$ after OGTT (mmol/l). The disposition index was calculated by multiplying the insulinogenic index and the Matsuda insulin sensitivity index, relating beta cell function to ambient insulin sensitivity. In addition, orally glucose-stimulated insulin release was reported as the BIGTT-acute insulin response (AIR) index. The BIGTT index applies information on sex and BMI combined with plasma glucose and serum insulin during an OGTT, to provide an index for AIR, and was calculated as reported previously [13].

Statistical analysis To test quantitative traits for differences between genotype groups, a general linear model was used, applying additive models for each variant and including adjustments for sex, age and with and without adjustment for BMI. Analyses were also performed using recessive and dominant models (see electronic supplementary material [ESM] Tables 1-4). The variants previously shown to be associated with WHR were also tested with adjustment for WHR, but this did not alter the results. All analyses were performed by RGui version 2.12.2 (R Development Core Team [1999]). Given the number of SNPs tested, we corrected for multiple testing with the Bonferroni correction leading to a statistical significance threshold of $0.05 / 32=$ 0.0016 . Associations with a $p$ value $<0.05$ but $>0.0016$ were considered to be nominally significant. All quantitative measures were logarithmically transformed before analysis.

The specific traits examined were fasting plasma glucose, fasting serum insulin, estimates of insulin sensitivity (HOMA-IR and ISI Matsuda), estimates of acute insulin response (BIGTT-AIR and insulinogenic index) and disposition index. Because of the known sexual dimorphism of WHR, analyses of variants previously associated with WHR were performed combined as well as for each sex. Interaction analyses were performed (see ESM Table 5). However, after adjustment for multiple testing, no significant interaction was found for the variants previously shown to be associated with WHR. Despite the lack of significant interactions, sex stratification is included, given the well-known biological sex differences of WHR. The sex-stratified analyses included 3,004 men and 3,035 women.

Statistical power analysis Statistical power for quantitative traits was estimated using simulations. An additive genetic model was used for both the simulation of the data and testing the data using a linear model. We used the allele frequencies of the 18 variants associated with BMI and 14 variants associated with WHR and a sample size of 6,039 individuals to estimate the effect size for each allele for which we had $80 \%$ statistical power to detect an association in the given quantitative trait. We carried out 5,000 simulations and applied a significance threshold of 0.0016 . We had $80 \%$ statistical power to detect an allelic difference of $0.9-1.2 \%$ in fasting plasma glucose, $4.3-5.7 \%$ in fasting serum insulin, 4.6-6.1\% in HOMA-IR, 4.4-5.8\% in disposition index, 3.1-4.1\% in BIGTT-AIR, and 0.27-0.51 absolute difference in BIGTT-sensitivity index, respectively.

Genotyping Genotyping of the 32 variants in 6,039 Danish individuals was performed using KASPar SNP genotyping (KBioscience, Hoddesdon, UK). The genotype distributions for 31 of the variants obeyed Hardy-Weinberg equilibrium in the population-based Inter99 study $(p>0.0016)$. The MTIF3 rs4771122 variant was slightly out of HardyWeinberg equilibrium $(p=0.0003)$. All 32 variants passed quality control with an average mismatch rate of $0.10 \%$ (maximum $0.66 \%$ ) and an average success rate of $96.3 \%$ (minimum 95.3\%) estimated from regenotyping 454 random duplicate samples. The number of genotyped SNPs in Inter99 differs slightly from SNP to SNP because of differences in genotyping success rate (95.3-97.1\%).

\section{Results}

We evaluated the relationship of 18 previously BMIassociated and 14 WHR-associated variants with glucoserelated metabolic traits in treatment-naive individuals from the population-based Inter99 study sample.

Metabolic associations of variants previously shown to relate to $B M I$ Of the variants associated with $\mathrm{BMI}$ (Table 1), the QPCTL rs2287019 BMI-associated C allele was associated with a higher insulinogenic index of $7.4 \%$ per risk allele $\left(p=7.2 \times 10^{-7}\right)$. This association remained after adjustment for BMI $\left(p=4.0 \times 10^{-7}\right)$. Moreover, the same variant was associated with a $5.6 \%$ increase in disposition index $\left(p=6.4 \times 10^{-5}\right)$, higher 30 min serum insulin levels $(p=$ $\left.1.4 \times 10^{-6}\right)$, higher AUC insulin $\left(p=2.1 \times 10^{-5}\right)$, higher $2 \mathrm{~h}$ plasma glucose levels $(p=0.0073)$, and higher AUC glucose $(p=0.0022)$ (data not shown). 


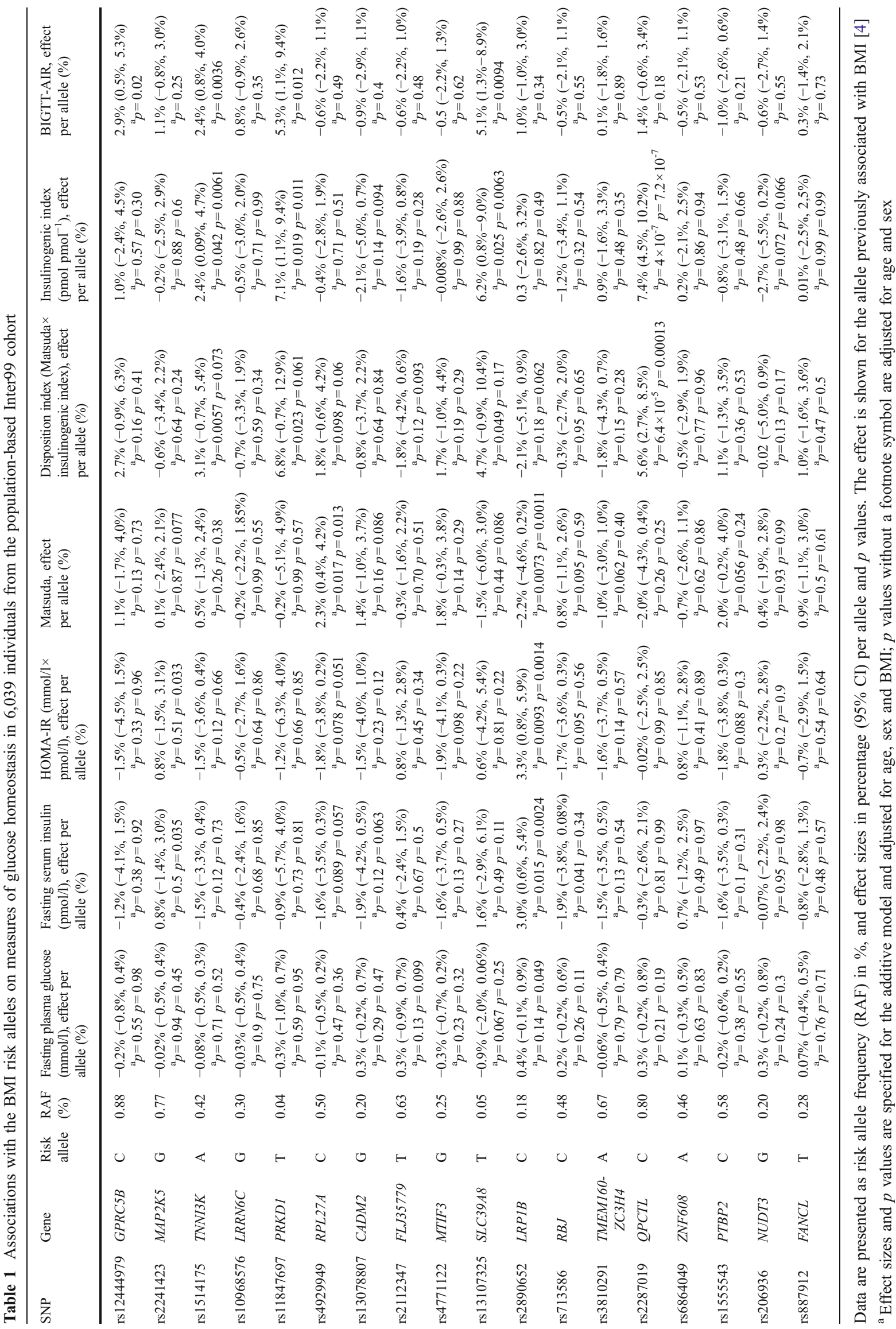


The $L R P 1 B$ rs2890652 BMI-increasing $\mathrm{C}$ allele showed association at a Bonferroni-corrected level, with a $3.3 \%$ decrease in insulin sensitivity per risk allele, as estimated by HOMA-IR $(p=0.0014)$, and a $2.2 \%$ reduction in Matsuda index $(p=0.0011)$ both when adjusted for sex and age. The association was slightly weaker in a model with adjustment for BMI (Table 1). rs2890652 C allele furthermore was associated nominally with an elevated fasting serum insulin level $(3.0 \% p=0.0024$, and $p=0.015$ after adjustment for BMI).

The remaining BMI-increasing alleles which only showed nominally significant effects on the examined traits included the TNNI3K rs1514175 A allele, the PRKD1 rs11847697 T allele and the SLC39A8 rs13107325 T allele. These alleles showed somewhat higher insulinogenic index (2.4\% $p=0.042,7.1 \% p=0.019$, and $6.2 \% p=0.025$, respectively), increased BIGTT-AIR (2.4\% $p=0.0036$, $5.3 \% p=0.012$, and $5.1 \% p=0.0094$, respectively), and increased disposition index $(3.1 \% p=0.0057,6.8 \% p=$ 0.023 , and $4.7 \% p=0.049$, respectively).

Metabolic associations of variants previously shown to relate to WHR Results for the WHR-associated variants are shown in Table 2 and ESM Tables 6 and 7. Of these variants, only the LYPLAL1/SLC30A10 rs4846567 $\mathrm{G}$ allele was associated at a Bonferroni-corrected level with improved insulin sensitivity, with a 3.5\% lower HOMA-IR per risk allele in a model adjusted for BMI $(p=0.0016)$. Furthermore, this variant was also nominally associated with decreased levels of fasting plasma glucose and fasting serum insulin $(0.5 \% p=0.036,3.0 \% p=0.0041$, respectively). When analyses were conducted in a sex-stratified manner, associations between this variant and the examined traits were found only in women in models adjusted for BMI (fasting plasma glucose, $0.9 \%[p=0.0026]$, fasting serum insulin, 4.2\% [ $p=0.0039]$, HOMA-IR, 5.2\% [ $p=0.00086]$, Matsuda index, 4.3\% [ $p=0.0086]$, and disposition index, $5.9 \%[p=0.0017])$. When calculated without adjustment for BMI, all the associations disappeared, except for disposition index (ESM Table 7).

Also, carriers of the VEGFA rs6905288 A allele presented a nominally significant $2.6 \%$ increase in insulin resistance per risk allele measured as HOMA-IR $(p=0.013)$, a $3.7 \%$ lower disposition index $(p=0.004)$, and a $2.4 \%$ increased fasting serum insulin level $(p=0.012)$. When stratified by sex, these associations were only found in women. The per allele effects of HOMA-IR $=3.7 \%(p=$ $0.00036)$, of fasting serum insulin $=3.5 \%(p=0.0004)$ and of Matsuda index $=4.0 \%\left(p=2 \times 10^{-4}\right)$ reached Bonferronicorrected significance levels when the analyses did not include adjustment for BMI (ESM Table 7).

A nominally increased level of fasting serum insulin of $2.8 \%$ per risk allele $(p=0.0034)$ and a $2.6 \%$ higher HOMA-
IR $(p=0.0098)$ per risk allele, indicating insulin resistance, was also found among carriers of the GRB14 rs10195252 $\mathrm{T}$ allele. However, these associations were only found in men (fasting serum insulin, 3.6\% $[p=0.0094]$ ) and (HOMA-IR. 3.4\% $[p=0.021]$ ) when sex-stratified analyses were performed.

Finally, the NFE2L3 rs1055144 T allele showed nominally significant lower levels of fasting plasma glucose of $0.7 \%$ per risk allele $(p=0.0097)$ and BIGTT-AIR of $2.8 \%$ $(p=0.0096)$. In sex-stratified analyses, a 1.0\% lower level of fasting plasma glucose was only found in men $(p=$ 0.0084); however, fasting serum insulin levels $(p=0.0095)$ and HOMA-IR $(p=0.021)$ were $4.5 \%$ and $4.2 \%$ higher, respectively, in women.

Combined effect of variants The combined effect of the BMI risk variants and WHR risk variants was examined on measures of glucose homeostasis in treatment-naive individuals from the Inter99 cohort. These analyses clearly showed that previously GWAS-identified variants were also associated with increased BMI (effect size, 0.01; 95\% CI $0.004,0.02 ; p=0.0014$, Fig. 1) and increased WHR (women: effect size, $0.006 ; 95 \%$ CI $0.003,0.009 ; p=$ 0.0005; men: effect size, $0.003 ; 95 \%$ CI 0.0009, 0.006; $p=$ 0.009 ; Figs 2 and 3, respectively) in the present study population. Analyses of the combined effect of BMI genetic variants showed association with several measures of glucose homeostasis; however, these results were not corrected for multiple testing and should therefore be validated in independent studies. Variants associated with increased BMI also associated with an increased disposition index $(0.03$ [0.007, 0.06], $p=0.01)$, which is a measure of the insulin secretion in relation to the levels of insulin resistance. Furthermore, the combined effect of the BMI variants associated with a $3 \%(95 \%$ CI $0.8,5)$ decrease in fasting serum insulin $(p=0.006)$ and increased insulin sensitivity: a 3\% $(95 \%$ CI $0.7,5)$ decrease in HOMA-IR $(p=0.01)$ and a $3 \%(95 \%$ CI $0.9,5)$ increase in Matsuda index $(p=0.005)$.

For WHR variants, association with measures of glucose homeostasis was only found in women. WHR variants associated with a $3 \%(0.4-5)$ increase in fasting serum insulin $(p=0.02)$, a $3 \%(0.7-6)$ increase in HOMA-IR $(p=0.01)$, and a $4 \%(1-7)$ decrease in disposition index $(p=0.008)$.

\section{Discussion}

By running large meta-analyses and extensive replication studies, the third wave of GWAS of adiposity-related traits has identified 14 variants associated with WHR and 18 variants associated with BMI. In the current random sample of the Danish adult population, we aimed to elucidate the 


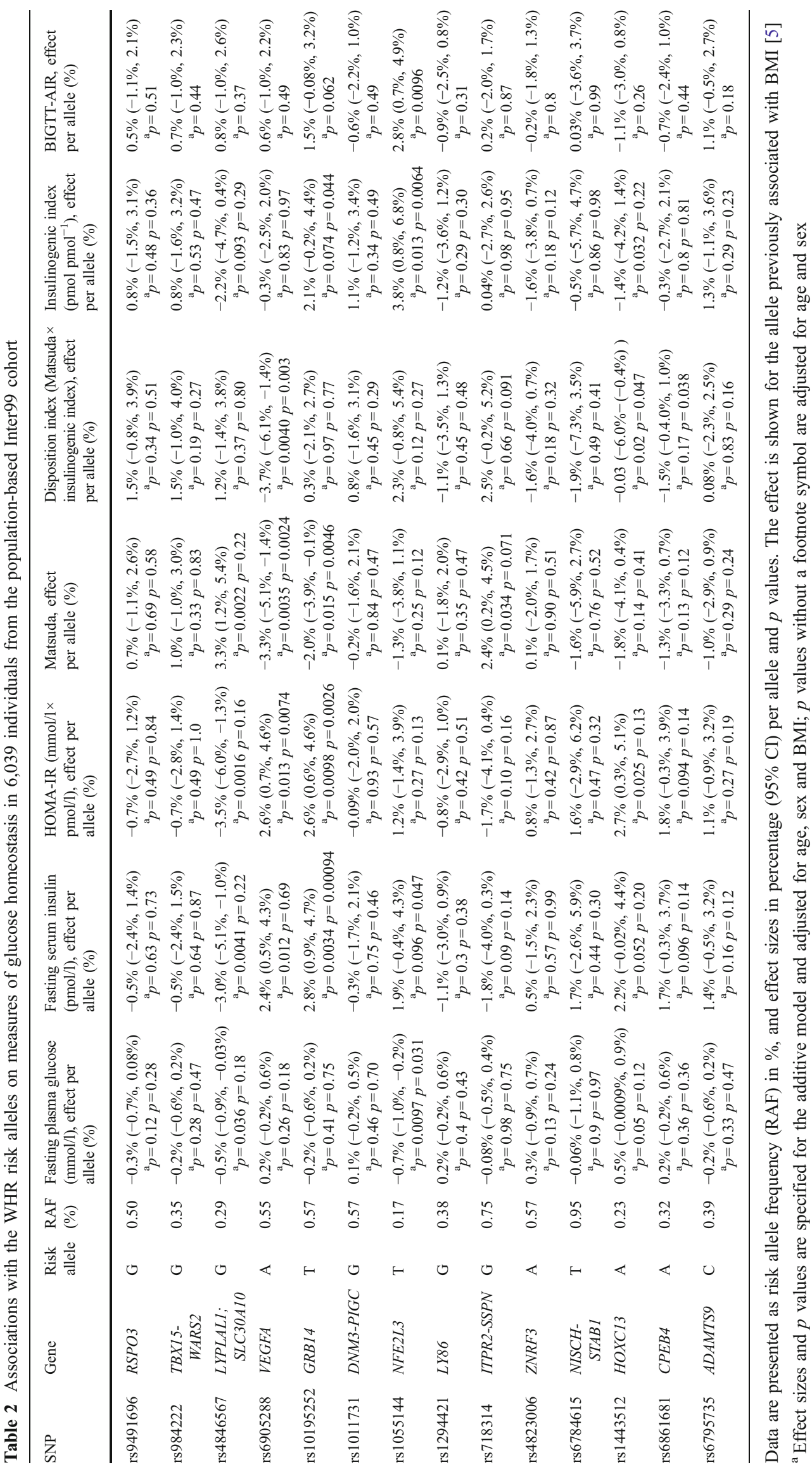




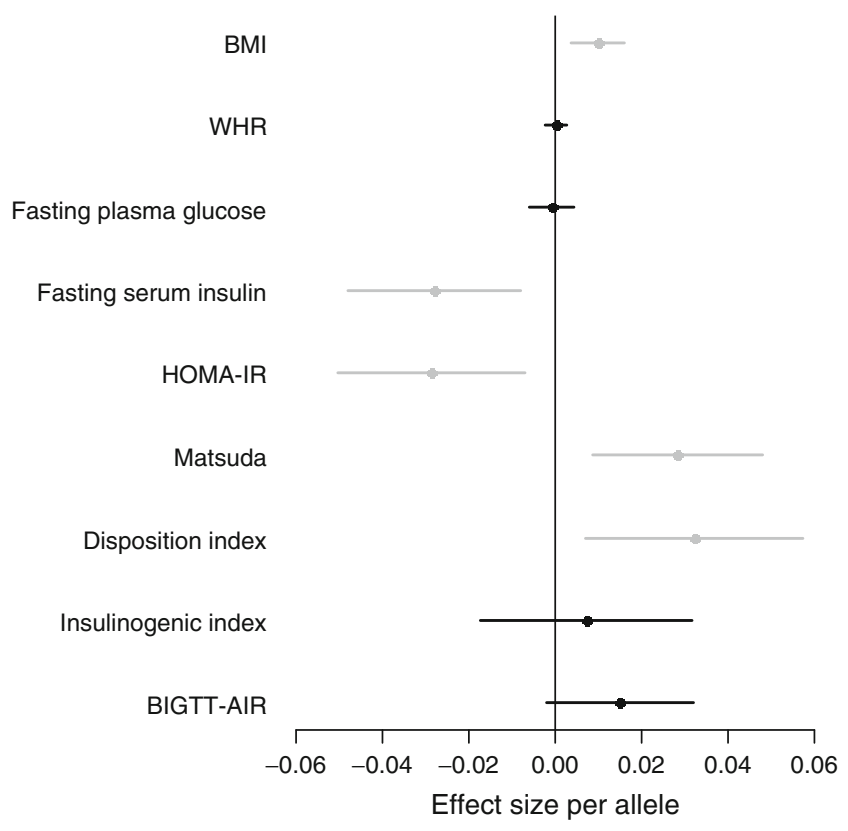

Fig. 1 Combined effects of BMI variants on measures of glucose homeostasis in 4,588 individuals of both sexes from the populationbased Inter99 cohort. Only individuals with a genotype for all investigated variants were included in the analyses of the combined effect $(1,451$ of the 6,039 individuals were excluded, leaving 4,588 individuals in this study). Black horizontal line, non-significant effect size; grey line, significant effect size $(p<0.05)$

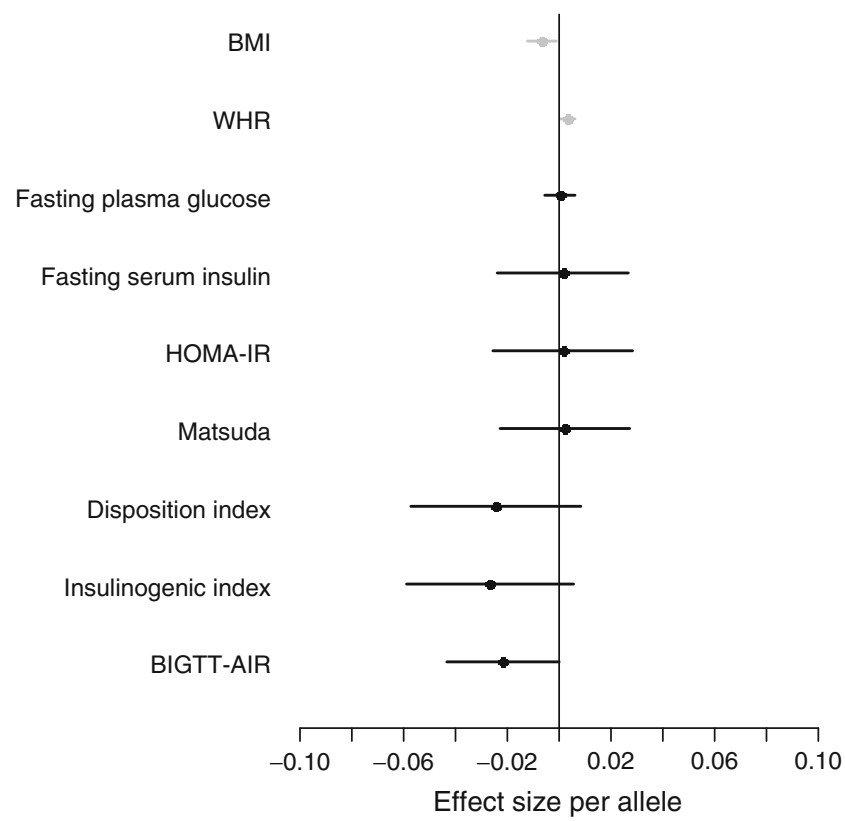

Fig. 2 Combined effects of WHR variants on measures of glucose homeostasis in 2,387 men from the population-based Inter99 cohort. Only men with a genotype for all investigated variants were included in the analyses of the combined effect (617 of the 3,004 individuals were excluded, leaving 2,387 individuals in this study). Black horizontal line, non-significant effect size; grey line, significant effect size $(p<0.05)$

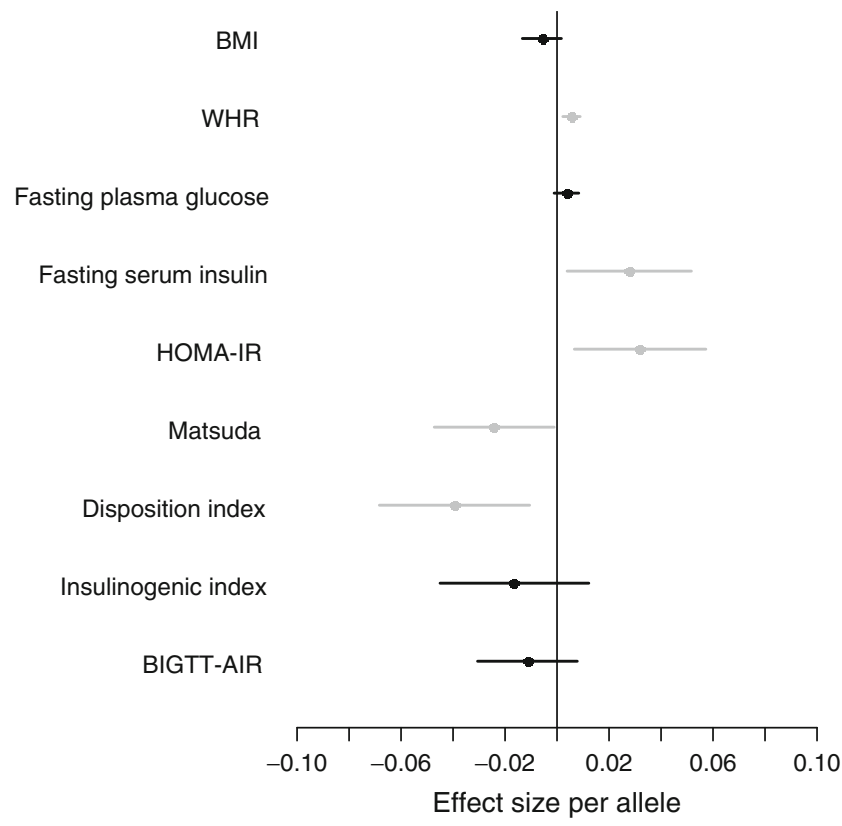

Fig. 3 Combined effects of WHR variants on measures of glucose homeostasis in 2,404 women from the population-based Inter99 cohort. Only women with a genotype for all investigated variants were included in the analyses of the combined effect (631 of the 3,035 individuals were excluded, leaving 2,404 individuals in this study). Black horizontal line, non-significant effect size; grey line, significant effect size $(p<0.05)$

impact of these variants on OGTT-based measures of insulin sensitivity and beta cell function in non-diabetic individuals, to possibly clarify the interplay between body composition and glucose homeostasis caused by these variants.

When the effect of the combined BMI risk variants and the combined WHR risk variants on measures of glucose homeostasis was evaluated, it appeared that these measures of body composition have different collective effects on glucose metabolism. Variants associating with an increased WHR appeared on the whole to reduce the ability of beta cells to produce insulin when estimated in relation to the ambient level of insulin resistance. The effects for WHR risk variants were further investigated in men and women separately, and the effects of WHR-associated loci on beta cell capacity were only seen in women. In contrast, the examined variants associating with increased BMI collectively led to an increased beta cell capacity. This may be a result of our study design, which included people who were overweight or obese but were still normoglycaemic.

The outcome of the single-variant analyses showed, however, that various adiposity-associated loci affect measures of insulin release and insulin sensitivity differently. For instance, we found that the QPCTL C allele was associated at a Bonferroni-corrected level with higher values of insulinogenic index and disposition index, whereas the $L R P 1 B$ rs $2890652 \mathrm{C}$ allele showed association 
with decreased insulin sensitivity. Both of these alleles represent loci that associate with increased levels of BMI.

The QPCTL rs2287019 is in high linkage disequilibrium with variants in GIPR, which have previously been shown to associate with beta cell function [14]. Supporting this observation, we found that the $Q P C T L$ variant associated with increased disposition index.

The BMI-associated variant in the LRP1B locus was associated with more than one glucose homeostatic measure after correction for multiple testing, whereas TNNI3K, PRKD1 and SLC39A8 variants after Bonferroni adjustment were associated with more homeostatic measures only at a nominal significance level. TNNI3K encodes a cardiac troponin I-interacting kinase with no known relation to glucose regulation. The same holds for PRKD1, which encodes an uncharacterised protein kinase. However, SLC39A8 is a member of the SLC39 family of solutecarrier genes, which show structural similarities to zinc transporters. Owing to the relationship between insulin secretion and zinc metabolism in the beta cell, the association with estimates of insulin release is not surprising [15]. LRPIB belongs to the LDL receptor gene family and therefore encodes a protein involved in metabolism [16].

On the whole, we did not observe any major effect of variants reported to associate with BMI on the fasting level of plasma glucose. This may indicate that, despite an increased requirement of insulin because of increased adiposity, the beta cells of variant carriers are capable of compensating for the relative and modest decrease in insulin sensitivity, which is in line with the observations from the combined analyses.

Of the variants associated with WHR, LYPLAL1/ SLC30A10 rs4846567 G allele associated after Bonferroni correction with lower HOMA-IR, indicating increased insulin sensitivity. In addition, decreased fasting levels of glucose and insulin and increased HOMA-IR were seen in women. These effects of variants that have been found to associate with increased WHR are obviously unexpected.

In contrast, there are several pieces of evidence for the insulin resistance of both male and female carriers of the VEGFA rs6905288 A allele, male carriers of the GRB14 T allele and female carriers of the NFE2L3 rs1055144 T allele, findings that can be expected from the associations of these risk alleles with increased WHR. However, only in female carriers did the association of the VEGFA rs6905288 A allele with insulin resistance reach statistical significance at a Bonferroni-corrected level.

All traits examined, including fasting plasma glucose, showed an association with one or more variants that were previously reported to associate with WHR.

We found that women, but not men, who carried the VEGFA rs6905288 A allele showed a Bonferroni-corrected association with decreased insulin sensitivity without adjustment for BMI. This association was still evident in models with BMI adjustment, pointing to a direct, adiposity-independent effect on insulin action. The protein encoded by VEGFA is known to be involved in adipogenesis, and raised levels of serum VEGFA have previously been shown to be associated with overweight and obesity $[17,18]$. A German study of 1,578 non-diabetic individuals reported that a variant (rs9472138 $\mathrm{T}$ allele) in VEGFA was associated with increased insulin secretion [19]. These findings make the VEGFA encoded protein a potential candidate in the pathogenesis of insulin resistance and adiposity.

Variants nominally associated with fasting plasma glucose included NFE2L3 and LYPLAL1. For NFE2L3, the signal was strongest in men, and this sex orientation is concordant with the GIANT study, where the variant showed one of the strongest associations with WHR in men [5]. In a previous study [20], the variant near LYPLAL1 was only found to associate with WHR in women, and the sex relatedness was confirmed in analysis of traits of glucose homeostasis. Because of our limited knowledge of the functions of the nearby genes, LYPLAL1 and NFE2L3, we are unable to explain the biological mechanisms behind these associations. The sex-specific effect of LYPLAL1 is in line with a previous meta-analysis of 16 GWAS $(n=38,580)$ and a follow-up of 70,689 individuals [20] in which variation in LYPLAL1 showed a sex-specific association with WHR. However, the T allele of rs 4846567 , which, in the previous study, associated with increased WHR, was associated with increased insulin sensitivity in genetic models adjusted for BMI in the present study. Also, the A allele of the variant located in the vicinity of VEGFA was associated with increased WHR in the GIANT study [5]; however, in the present study, the same variant was associated with increased insulin sensitivity independently of BMI. We have no obvious interpretation of this finding other than that, in the present study, we excluded individuals with known diabetes and by doing so may have selected individuals possessing adaptive mechanisms to cope with the projected adiposity-elicited impairment of insulin sensitivity.

We note that a limitation of our explorative study is the fact that we only corrected for the number of variants analysed, without correcting for the different, but interrelated, metabolic traits that we investigated. This limitation will obviously increase the risk of type I errors.

In conclusion, our correlative findings from analysing single-locus data suggest that some variation in validated BMI- and WHR-associated loci are related to either increased or decreased insulin sensitivity and therefore potentially to metabolically healthy or metabolically unhealthy subsets of obesity. However, when analysed 
collectively, variants associated with an increased WHR appeared to reduce insulin release, and variants associated with increased BMI appeared collectively to lead to an increase in insulin release. Therefore, more in-depth physiological studies are needed to disentangle how single adiposity-associated loci and combined adiposityassociated loci, respectively, affect insulin sensitivity and insulin release.

Acknowledgements The authors wish to thank A. Forman, T. Lorentzen and M. Stendal for technical assistance, G. Lademann for secretarial support, A. Nielsen and P. Sandbeck for data management, and $\mathrm{M}$. Kristensen for grant management. This work received support from Lundbeck Foundation Centre for Applied Medical Genomics in Personalized Disease Prediction, Prevention and Care (LuCAMP, www.lucamp.org) and The Danish Obesity Research Centre (DanORC, www.danorc.dk), which is supported by The Danish Council for Strategic Research (Grant No 2101-06-0005). The Inter99 was initiated by T. Jørgensen (PI), K. Borch-Johnsen (coPI), H. Ibsen and T. F. Thomsen. The steering committee comprises the former two and C. Pisinger.

Contribution statement $\mathrm{KSB}, \mathrm{APG}, \mathrm{NG}, \mathrm{JMJ}$ and OP contributed to the conception and design of the study. KSB, APG, NG, JMJ, CHS, DRW, TJ, SM, TH and OP contributed to analysis and interpretation of data. KSB, APG, NG, JMJ, CHS, DRW, TJ, SM, TH and OP contributed to the drafting the article or revising it critically for important intellectual content. KSB, APG, NG, JMJ, CHS, DRW, TJ, $\mathrm{SM}, \mathrm{TH}$ and $\mathrm{OP}$ have approved the final version for the paper to be published.

Duality of interest The authors declare that there is no duality of interest associated with this manuscript.

\section{References}

1. Herrera BM, Lindgren CM (2010) The genetics of obesity. Curr Diab Rep 10:498-505

2. Frayling TM, Timpson NJ, Weedon MN et al (2007) A common variant in the FTO gene is associated with body mass index and predisposes to childhood and adult obesity. Science 316:889-894

3. Loos RJ, Lindgren CM, Li S et al (2008) Common variants near MC4R are associated with fat mass, weight and risk of obesity. Nat Genet 40:768-775

4. Speliotes EK, Willer CJ, Berndt SI et al (2010) Association analyses of 249,796 individuals reveal 18 new loci associated with body mass index. Nat Genet 42:937-948
5. Heid IM, Jackson AU, Randall JC et al (2010) Meta-analysis identifies 13 new loci associated with waist-hip ratio and reveals sexual dimorphism in the genetic basis of fat distribution. Nat Genet 42:949-960

6. Price GM, Uauy R, Breeze E, Bulpitt CJ, Fletcher AE (2006) Weight, shape, and mortality risk in older persons: elevated waisthip ratio, not high body mass index, is associated with a greater risk of death. Am J Clin Nutr 84:449-460

7. Hofker M, Wijmenga C (2009) A supersized list of obesity genes. Nat Genet 41:139-140

8. Thorleifsson G, Walters GB, Gudbjartsson DF et al (2009) Genome-wide association yields new sequence variants at seven loci that associate with measures of obesity. Nat Genet 41:18-24

9. Willer CJ, Speliotes EK, Loos RJ et al (2009) Six new loci associated with body mass index highlight a neuronal influence on body weight regulation. Nat Genet 41:25-34

10. Jorgensen T, Borch-Johnsen K, Thomsen TF, Ibsen H, Glumer C, Pisinger C (2003) A randomized non-pharmacological intervention study for prevention of ischaemic heart disease: baseline results Inter99. Eur J Cardiovasc Prev Rehabil 10:377-386

11. Matthews DR, Hosker JP, Rudenski AS, Naylor BA, Treacher DF, Turner RC (1985) Homeostasis model assessment: insulin resistance and beta-cell function from fasting plasma glucose and insulin concentrations in man. Diabetologia 28:412-419

12. Matsuda M, DeFronzo RA (1999) Insulin sensitivity indices obtained from oral glucose tolerance testing: comparison with the euglycemic insulin clamp. Diabetes Care 22:1462-1470

13. Hansen T, Drivsholm T, Urhammer SA et al (2007) The BIGTT test: a novel test for simultaneous measurement of pancreatic betacell function, insulin sensitivity, and glucose tolerance. Diabetes Care 30:257-262

14. Saxena R, Hivert MF, Langenberg $C$ et al (2010) Genetic variation in GIPR influences the glucose and insulin responses to an oral glucose challenge. Nat Genet 42:142-148

15. Jansen J, Karges W, Rink L (2009) Zinc and diabetes-clinical links and molecular mechanisms. J Nutr Biochem 20:399-417

16. May P, Woldt E, Matz RL, Boucher P (2007) The LDL receptorrelated protein (LRP) family: an old family of proteins with new physiological functions. Ann Med 39:219-228

17. Silha JV, Krsek M, Sucharda P, Murphy LJ (2005) Angiogenic factors are elevated in overweight and obese individuals. Int $\mathrm{J}$ Obes (Lond) 29:1308-1314

18. Garcia de la Torre N, Rubio MA, Bordiu E et al (2008) Effects of weight loss after bariatric surgery for morbid obesity on vascular endothelial growth factor-A, adipocytokines, and insulin. J Clin Endocrinol Metab 93:4276-4281

19. Staiger H, Machicao F, Kantartzis K et al (2008) Novel metaanalysis-derived type 2 diabetes risk loci do not determine prediabetic phenotypes. PLoS One 3:e3019

20. Lindgren CM, Heid IM, Randall JC et al (2009) Genome-wide association scan meta-analysis identifies three loci influencing adiposity and fat distribution. PLoS Genet 5:e1000508 